\title{
Network analysis and transcriptome profiling in peripheral blood mononuclear cells of patients with rheumatoid arthritis
}

\author{
YAN LONG ${ }^{1,2}$, JIAN LIU $^{2,3}$, HUI JIANG ${ }^{2}$, LING XIN $^{2}$, LEI WAN ${ }^{3}$, YUE SUN $^{3}$, PINGHENG ZHANG $^{4}$, \\ JIANTING WEN ${ }^{2}$, DAN HUANG ${ }^{2}$, YANQIU SUN ${ }^{2}$, YING ZHANG ${ }^{2}$, BINGXI BAO $^{2}$ and GUANGHAN SUN ${ }^{2}$ \\ ${ }^{1}$ Department of Graduate, Anhui University of Chinese Medicine; ${ }^{2}$ Laboratory for Rheumatism, \\ Institute of Rheumatology, The First Affiliated Hospital of Anhui University of Chinese Medicine, \\ Hefei, Anhui 230011; ${ }^{3}$ Department of Rheumatology and Immunology, The First Affiliated Hospital \\ of Anhui University of Chinese Medicine, Hefei, Anhui 230038; ${ }^{4}$ Department of Chinese Medicine, \\ Zhujiang Hospital, Southern Medical University, Guangzhou, Guangdong 510280, P.R. China
}

Received June 15, 2020; Accepted October 6, 2020

DOI: 10.3892/etm.2020.9601

\begin{abstract}
The present study aimed to investigate the differential expression of long non-coding RNAs (lncRNAs) in rheumatoid arthritis (RA). High-throughput gene sequencing technology was used to detect the expression of lncRNA and mRNA in three patients with RA (RA group) and normal controls (NC group). A Bioinformatics analysis was used to assess the effects of differentially expressed mRNAs on signaling pathways and biological functions. The selected dysregulated lncRNAs were verified by reverse transcription-quantitative (RT-q)PCR in the peripheral blood mononuclear cells (PBMCs) of patients with RA and age- and sex-matched controls. A correlation analysis was used to analyze the relationship between lncRNAs and clinical indexes. From the lncRNA sequencing data, significantly differentially expressed lncRNAs between the RA and NC groups were identified by a fold change $\geq 2$ and $P<0.05$. Gene Ontology and Kyoto Encyclopedia of Genes and Genomes analysis suggested that the differentially expressed mRNAs were mainly involved in organelle composition, intracellular regulation, signaling pathways, cancer, virus and inflammation. A total of four of these lncRNAs were confirmed by RT-qPCR to be
\end{abstract}

Correspondence to: Professor Jian Liu, Laboratory for Rheumatism, Institute of Rheumatology, The First Affiliated Hospital of Anhui University of Chinese Medicine, 117 Meishan Road, Shushan, Hefei, Anhui 230011, P.R. China

E-mail: liujianahzy@126.com

Abbreviations: C3, complement 3; anti-CCP, anti-cyclic citrullinated peptide antibody; hs-CRP, high-sensitivity C-reactive protein; DAS-28 score, 28-joint count rheumatoid arthritis disease activity score; ESR, erythrocyte sedimentation rate; NC, normal control; RA, rheumatoid arthritis; IgA, immunoglobulin A; lncRNA, long non-coding RNA; RT-PCR, reverse transcription-PCR; RF, rheumatoid factor; VAS, visual analogue scale

Key words: rheumatoid arthritis, long non-coding RNA, mRNA, sequencing, patient perception index significantly differentially expressed (LINC00304, MIR503HG, LINC01504 and FAM95B1). Through the correlation analysis, it was confirmed that there was a strong correlation between these lncRNAs and clinical laboratory indicators and indexes such as course of disease, arthrocele and joint tenderness. Overall, the present results suggested that the expression levels of LINC00304,MIR503HG,LINC01504 and FAM95B1 in PBMCs from patients with RA may serve as potential biomarkers for RA diagnosis, influencing the occurrence and progress of RA.

\section{Introduction}

Rheumatoid arthritis (RA) is an autoimmune disease of the human body that is characterized by progressive destruction of the peripheral joint and its surrounding tissues (1). The global incidence of RA has been estimated to be $0.5-1 \%$ (2). The pathogenesis of RA is complex and may be caused by unknown antigens that are sensitive to certain inherited factors. Genetic, epigenetic and environmental factors may contribute to disease susceptibility and progression. The factors (that require confirmation), such as viruses, sex hormones and neurodevelopmental state, are closely related (3). Due to its numerous complications and high disability rate, RA has a significant impact on affected individuals, their families and society, and places an emotional and financial burden on them (4). Therefore, it is of great significance to explore the pathogenesis of RA and discover biomarkers and therapeutic targets for reducing the disability rate of RA and improving the quality of life of patients.

Long non-coding RNAs (lncRNAs) are $>200$ nucleotides in length and account for $98 \%$ of the ncRNA in the transcribed human genome (5). Certain studies have indicated that lncRNAs have an indispensable role in the pathogenesis of RA $(6,7)$. For instance, a novel type of lncRNA, C5T1lncRNA, transcribed from a region between the TNF receptor-associated factor 1 (TRAF1) and TRAF1-complement component 5 (TRAF1-C5) genes, has been reported to be mainly expressed in the nucleus. A positive correlation between C5T1lncRNA and the expression of TRAF1-C5 was identified in synovial fibroblasts and peripheral blood mononuclear cells (PBMCs) of patients with RA. C5T1lncRNA is able to participate in the pathogenesis of 
RA by regulating the transcription of the TRAF1-C5 gene in the same genomic region (8).

Although the role of lncRNAs in RA has been increasingly investigated, the current understanding of the function and regulatory mechanisms of lncRNAs in the development and progression of RA remains limited. In particular, the expression of lncRNAs and mRNAs in PBMCs of patients with RA and the relationship between IncRNAs and clinical indexes remain to be determined.

In the present study, after comparing $3 \mathrm{RA}$ samples with 3 negative control (NC) samples to characterize the lncRNA expression profile in PBMCs, 10 RA samples and $10 \mathrm{NC}$ samples were selected to evaluate the expression of lncRNA by reverse transcription-quantitative (RT-q)PCR. The correlations between the IncRNAs and the RA disease activity index, disease activity score (DAS) including a 28-joint count rheumatoid arthritis DAS (DAS-28) and the 36-item short-form survey (SF-36) score $(9,10)$, were analyzed. The results indicated that differentially expressed lncRNAs may be involved in the pathogenesis of RA, suggesting that they may have potential as novel biomarkers for the diagnosis and prognosis of RA. In the future, experiments with a larger sample size will be required to verify the present results and to clarify the regulatory mechanisms of the roles of lncRNAs in patients with RA.

\section{Materials and methods}

Patients and biochemical measurements. A total of 10 patients with RA were selected from the Department of Rheumatology and Immunology, the First Affiliated Hospital of Anhui University of Chinese Medicine (FAHAUCM; Hefei, China) between June 2019 and July 2019. The cohort comprised 9 females and 1 male with a mean age of 43.7 years (range, 27 to 68 years). Furthermore, 10 normal controls (NCs) were recruited, who were age and sex-matched with the case group and who received a routine physical examination at the Department of Health of the FAHAUCM. All patients with RA fulfilled the new classification criteria of RA proposed by the American Rheumatology Society and the European Union against Rheumatism in 2010 (11). Patients who had serious heart, liver or kidney diseases, mental disorders and pregnant or lactating females were excluded. Informed consent was obtained from all participants, including the RA group and NC group, prior to initiation of the study and it was approved by the Ethics Committee of the FAHAUCM. It was performed in compliance with the recommendations of the Declaration of Helsinki.

For patients with RA, disease activity was determined using the disease activity SF-36 scale and the DAS-28 scoring system based on a questionnaire survey (10). This provided an absolute number reflecting disease activity from four items in DAS-28, including the number of arthrocele and tender joints, the visual analogue scale and the erythrocyte sedimentation rate (ESR) in the first hour (12). ESR, high-sensitivity C-reactive protein (CRP), rheumatoid factor (RF), anti-cyclic citrullinated peptide antibody, immunoglobulin A (IgA), IgG, IgM, C3 and C4 were measured enzymatically using an autoanalyzer (Hitachi 747; Hitachi). Patients with values of $>8 \mathrm{mg} / \mathrm{ml}$ for CRP and $\mathrm{RF}>20 \mathrm{IU} / \mathrm{ml}$ were considered positive for $\mathrm{RA}$.

PBMC preparation and total RNA extraction. From each donor, 5-ml blood samples collected immediately were added to $7.5 \mathrm{ml}$ Ficoll-Paque plus (Cytiva) and centrifuged at 1,2000 g for $15 \mathrm{~min}\left(2-8^{\circ} \mathrm{C}\right)$. It has been proposed that the intermediate flocs should be washed and collected twice with $9 \mathrm{ml}$ PBD. The intermediate flocs were collected twice by centrifugation at 7,500 gx5 $\min \left(2-8^{\circ} \mathrm{C}\right)$. Subsequently, total RNA was extracted from the freshly obtained PBMCs using TRIzol reagent (Takara Bio, Inc.) according to the manufacturer's protocol.

lncRNA and mRNA sequencing (seq). The quality of all participants' RNA (including participants who are sequenced first and participants who are verified later) were evaluated by a nanometer photometer spectrophotometer(IMPLEN). The Qubit RNA Assay Kit with the Qubit 2.0 Fluorometer (Thermo Fisher Scientific, Inc.) and an Agilent Bioanalyzer 2100 (Agilent Technologies, Inc.) were used to determine the RNA concentration and examine the RNA integrity, respectively. Subsequently, the RNA library was constructed using a total amount of $3 \mu \mathrm{g}$ of RNA each sample, and its RNA integrity number was $>7.0$. Ribosomal RNA (rRNA) was removed by the Epicentre Ribo-zero rRNA Removal kit (Epicentre; Illumina, Inc.) according to the manufacturer's protocol. Afterwards, the strand-specific sequencing libraries was obtained by the New England Biolabs (NEB) Next Ultra Directional RNA Library Prep Kit for Illumina (NEB) using the dUTP method. The RNA-seq assay was accomplished on an Illumina Hiseq 2000 platform (Illumina, Inc.) and 100 bp paired-end reads were obtained. The preparation of the total transcriptome libraries was completed by YuXi Bioinformatics Corp.

Verification of significantly upregulated lncRNAs. PBMCs were isolated immediately after 5-ml blood samples were collected from each donor. PBMCs from 10 patients with RA and $10 \mathrm{NCs}$ were used for validation of six lncRNAs by RT-qPCR. Total RNA was obtained from the PBMCs with TRIzol (Thermo Fisher Scientific, Inc.) and was used for synthesis of complementary DNA with the ReverTraAc real-time qPCR kit (Takara Bio, Inc.) (13). The amplification conditions were as follows: Pre-denaturation at $95^{\circ} \mathrm{C}$ for $10 \mathrm{~min}$; denaturation at $95^{\circ} \mathrm{C}$ for $15 \mathrm{sec}$, annealing at $60^{\circ} \mathrm{C}$ for $30 \mathrm{sec}$, and extension for $120 \mathrm{sec}$. A total of 25 cycles and 30 cycles were used to amplify the 500 bp fragment (14) The primer sequences are provided in Table I. Divergent primer design refers to Panda and Gorospe. To synthesize cDNA from total RNA, Prime-Script Master Mix (Takara Bio, Inc.) was used $\left(60^{\circ} \mathrm{C}\right.$ for $\left.30 \mathrm{~min}\right)$. The relative expression of lncRNA was subsequently detected using the TB Green Premix Ex Taq II (Tli RNaseH Plus; Takara Bio, Inc.) with $\beta$-actin as an internal control (15). The relative expression levels were quantified from three independent experiments by the $2^{-\Delta \Delta \mathrm{Cq}}$ method (16).

Functional group analysis. Analysis of the biological functions and signaling pathways of the abnormally expressed mRNAs was performed by utilizing the Gene Ontology (GO; www.genontology.org) and Kyoto Encyclopedia of Genes and Genomes (KEGG; www.genome.ad.jp/KEGG) databases $(17,18) . \mathrm{P}<0.05$ was considered to indicate a statistically significant difference. The top 20 significantly differentially expressed genes were subjected to GO functional enrichment analysis. On the abscissa, the functional terms in the three basic categories of GO (biological process, cellular component and molecular function) were presented. The enrichment of KEGG was calculated in a similar manner as the GO analysis. 
Table I. Specific primers used for quantitative PCR analysis.

\begin{tabular}{|c|c|c|}
\hline Gene name & Sequence $\left(5^{\prime}-3^{\prime}\right)$ & Product length (bp) \\
\hline \multirow[t]{2}{*}{ LINC01504 } & F:TTGGCTAACGGAGTTTTGCT & 146 \\
\hline & R:CTTCTGAGGCCTGGATCTTG & \\
\hline \multirow[t]{2}{*}{ LINC00968 } & F:GCCCAGTTGACAGGAAATGT & 182 \\
\hline & R:TTGGTTCTCAATGGGATGGT & \\
\hline \multirow[t]{2}{*}{ FAM95B1 } & F:GGAGCTCAGTGCCCTCATAG & 141 \\
\hline & R:GCTCCAGGATGATGGTGTCT & \\
\hline \multirow[t]{2}{*}{ MIR503HG } & F:CCCCCAACAAAGGAACACTA & 142 \\
\hline & R:ACTTGGGTGGTTTTCAATGC & \\
\hline \multirow[t]{2}{*}{ LINC00304 } & F:CCGTCCAAGAGCAAAGCTAC & 143 \\
\hline & R:GGCATCAGGCAAAATCAAGT & \\
\hline \multirow[t]{2}{*}{ LINC01146 } & F:ATTCAGCCAACCAACTGAGG & 147 \\
\hline & R:TCACAGGTTCTGTGGGTCAA & \\
\hline \multirow[t]{2}{*}{ GAPDH } & F:GGAGCGAGATCCCTCCAAAAT & 205 \\
\hline & R:GGCTGTTGTCATACTTCTCATGG & \\
\hline
\end{tabular}

F, forward; R, reverse.

Statistical analysis. The statistical significance of differences was conveniently estimated by a t-test of the RNA-seq data. lncRNAs (up- or downregulated) with fold changes (FC) $\geq 2$ and $\mathrm{P}<0.05$ were selected as being significantly differentially expressed and the false discovery rate was calculated to correct the P-value. Values are expressed as the mean \pm standard deviation. The groups were compared to evaluate the statistical significance using the Mann-Whitney U test, Student's t-test, Wilcoxon signed-rank test or Chi-square test, as appropriate. GraphPad Prism 7.0 (GraphPad Software, Inc.) and SPSS (version 22.0; IBM Corp.) were used to analyze all statistical data. $\mathrm{P}<0.05$ was considered to indicate statistical significance.

\section{Results}

Clinical and biochemical features of the included individuals. As presented in Table II, there were no obvious differences between the patients with RA and healthy subjects in terms of sex and age. The baseline parameters of the two groups were consistent and comparable.

IncRNA expression profiles of patients with $R A$. In the present study, 231 upregulated and 110 downregulated lncRNAs were identified as differentially expressed in patients with RA compared with the controls. Information on the top 6 up- or downregulated lncRNAs is provided in Table III.

Analysis of differentially expressed lncRNAs and mRNAs. From the IncRNA expression profiles, differentially expressed IncRNAs between the RA group and the NC group were identified, consisting of 231 upregulated and 110 downregulated lncRNAs, according to the filtering criteria of $\mathrm{P}<0.05$ and $F C \geq 2$. Graphical presentations of the differentially expressed lncRNAs are provided in Fig. 1. Scatter plots and volcano plots were used to display the lncRNAs based on their expression levels compared between samples (Fig. 1A and C). In order to
Table II. Clinical characteristics of the study population.

\begin{tabular}{lccc}
\hline Index & RA $(\mathrm{n}=10)$ & Control $(\mathrm{n}=10)$ & P-value \\
\hline Sex $(\mathrm{M} / \mathrm{F})$ & $1: 9$ & $1: 9$ & 1 \\
Age $($ years $)$ & $45.9 \pm 11.80$ & $43.7 \pm 7.99$ & 0.637 \\
ESR $(\mathrm{mm} / \mathrm{h})$ & $38 \pm 27.48$ & $\mathrm{NA}$ & $\mathrm{NA}$ \\
$\mathrm{Hs}-\mathrm{CRP}(\mathrm{mg} / \mathrm{l})$ & $23.40 \pm 36.40$ & $\mathrm{NA}$ & $\mathrm{NA}$ \\
$\mathrm{RF}(\mathrm{U} / \mathrm{ml})$ & $109.48 \pm 82.34$ & $\mathrm{NA}$ & NA \\
Anti-CCP $(\mathrm{U} / \mathrm{ml})$ & $164.75 \pm 226.34$ & NA & NA \\
IgA $(\mathrm{g} / \mathrm{l})$ & $2.53 \pm 0.57$ & NA & NA \\
IgG $(\mathrm{g} / \mathrm{l})$ & $13.88 \pm 3.40$ & NA & NA \\
IgM $(\mathrm{g} / \mathrm{l})$ & $1.48 \pm 0.61$ & NA & NA \\
C3 $(\mathrm{g} / \mathrm{l})$ & $1.23 \pm 0.27$ & NA & NA \\
C4 $(\mathrm{g} / \mathrm{l})$ & $0.34 \pm 0.23$ & NA & NA \\
DAS-28 score & $6.80 \pm 0.98$ & NA & NA \\
VAS score & $6.95 \pm 0.94$ & NA & NA \\
SAS score & $56.4 \pm 4.88$ & NA & NA \\
SDS score & $56.6 \pm 6.07$ & NA & NA \\
SF-36 score & $1302.1 \pm 14.99$ & NA & NA \\
\hline
\end{tabular}

Values are expressed as $\mathrm{n}$ or the mean \pm standard deviation. NA, not available; M, male; F, female; RA, rheumatoid arthritis; C3, complement 3; anti-CCP, anti-cyclic citrullinated peptide antibody; hs-CRP, high-sensitivity C-reactive protein; DAS-28 score, 28-joint count rheumatoid arthritis disease activity score; ESR, erythrocyte sedimentation rate; $\mathrm{NC}$, normal control; RA, rheumatoid arthritis; IgA, immunoglobulin A; lncRNA, long non-coding RNA; RT-PCR, reverse transcription-PCR; RF, rheumatoid factor; VAS, visual analogue scale; SAS, self-rating anxiety scale; SDS, self-rating depression scale; SF-36, 36-item short-form survey.

further reveal the differences of the lncRNAs between samples, a hierarchical clustering analysis was applied, as presented in 
Table III. Information on the top six up- or down-regulated lncRNAs in patients with rheumatoid arthritis compared with healthy controls.

\begin{tabular}{lcccc}
\hline IncRNAs & P-value & Fold change & Direction of regulation & Gene symbol \\
\hline ENSG00000246430 & 0.001366 & 2.747691448 & Up & LINC00968 \\
ENSG00000223749 & 0.002933 & 2.532423677 & Up & MIR503HG \\
ENSG00000225434 & 0.000301 & -3.210084216 & Down & LINC01504 \\
ENSG00000223839 & 0.002506 & -2.536755148 & Down & FAM95B1 \\
ENSG00000180422 & 0.003974 & -2.794683328 & Down & LINC00304 \\
ENSG00000258867 & 0.004095 & -2.478197951 & Down & LINC01146
\end{tabular}

lncRNA, long non-coding RNA.
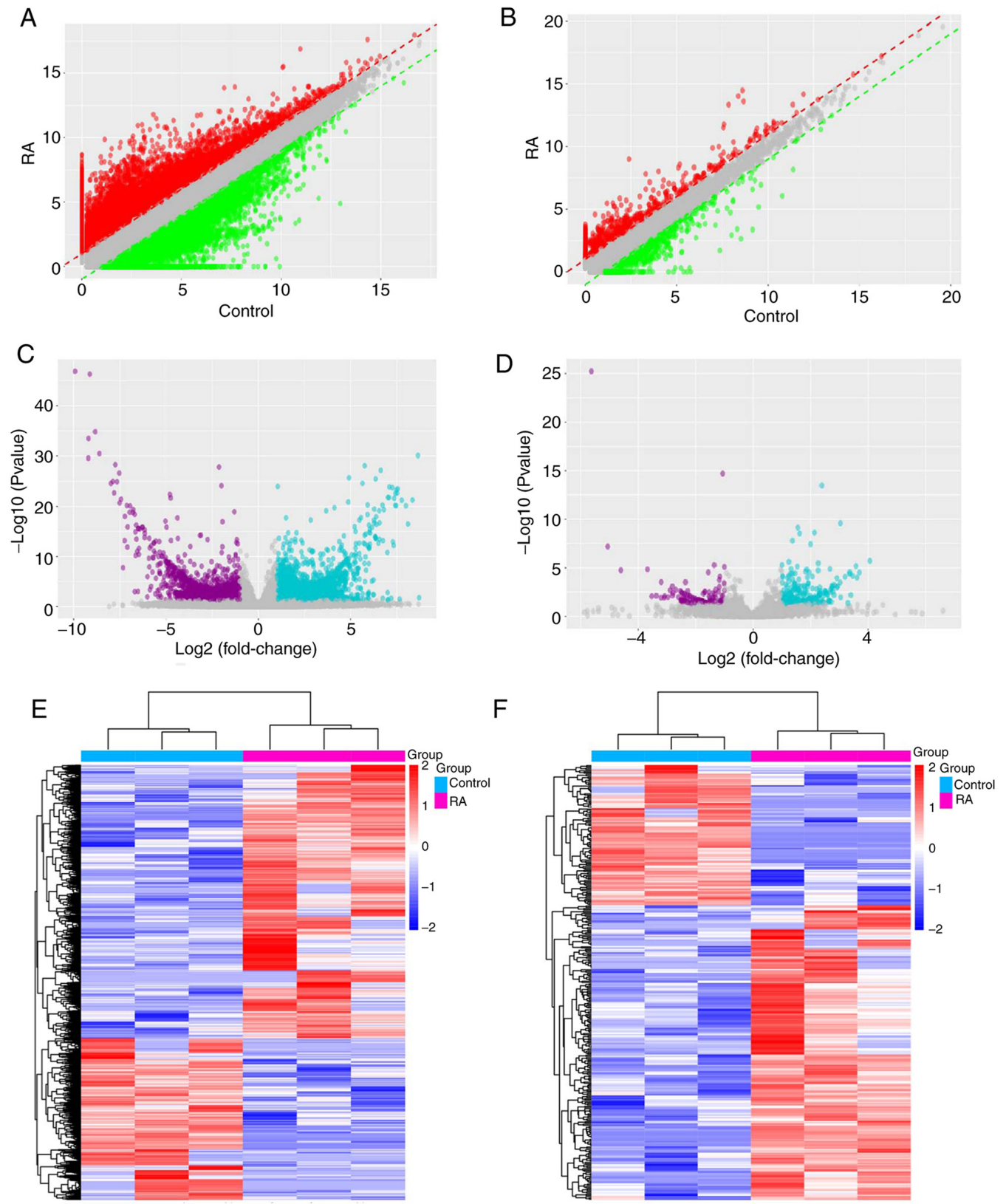

Figure 1. (A and B) Dot plots comparing the expression of RNAs between the RA and control groups. The dots above the upper and lower green lines indicate $>2.0$-fold changes in mRNAs (A) and lncRNAs (B) between the two groups. (C and D) In the volcano plots, purple represents a significant increase, blue represents a significant decline and gray represents no significant change in mRNAs (C) and lncRNAs (D). (E and F) Heat maps of differentially expressed RNAs. Each row represents an RNA and each column represents a sample. Blue indicates downregulated and red indicates upregulated (E) mRNAs and (F) lncRNAs. lncRNA, long non-coding RNA; RA, rheumatoid arthritis. 


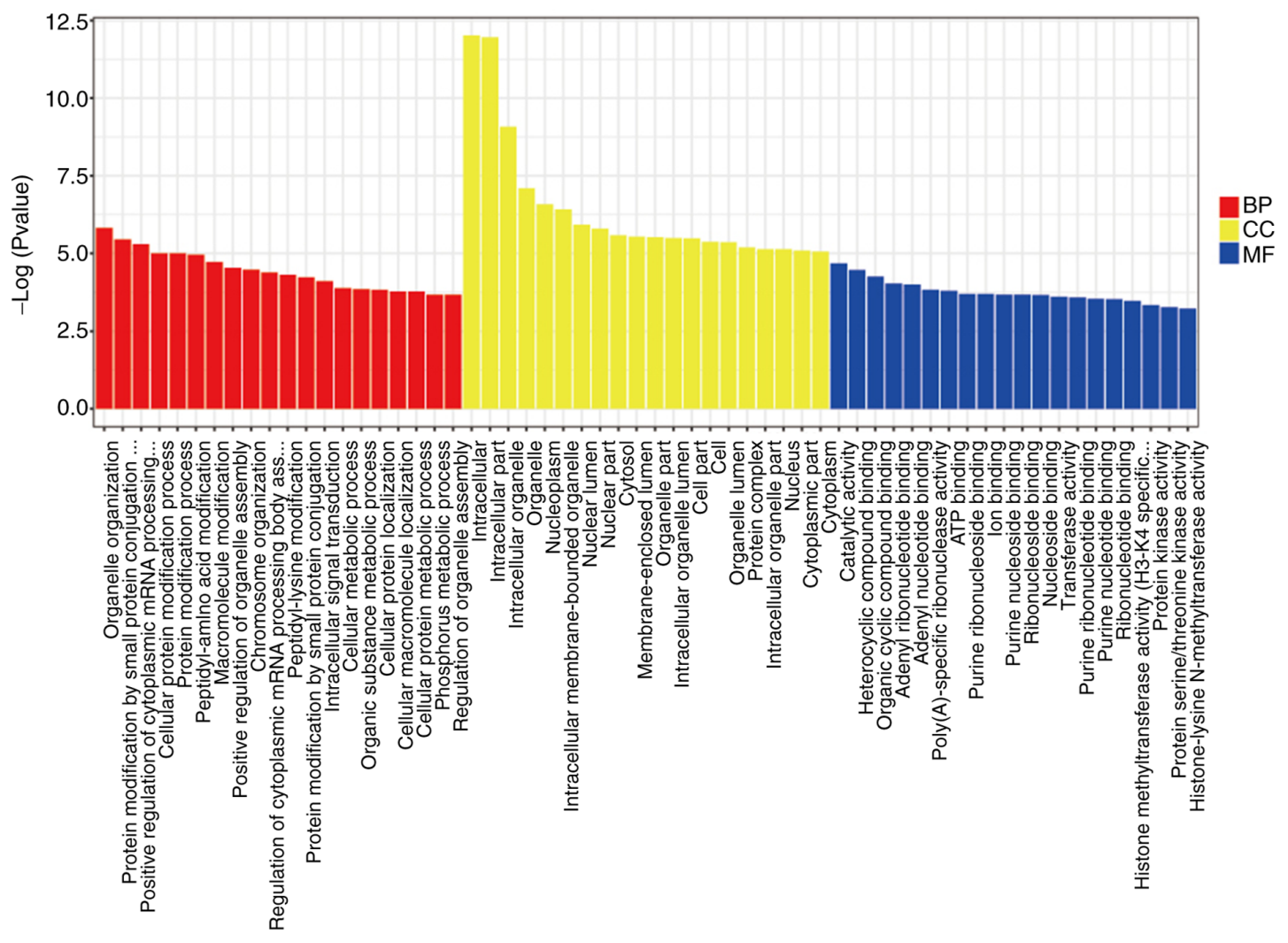

GO term

Figure 2. Biological function analysis of differentially expressed mRNAs. Red, yellow and blue bars represent GO terms in the categories BP, CC and MF, respectively. The number of genes annotated to a term is presented on the ordinate. The vertical length of the column indicates the enrichment degree, with long columns representing high degrees of enrichment. GO, gene ontology; BP, biological process; CC, cellular component; MF, molecular function.

Fig. 1E, and it was indicated that there was a general difference between patients and controls in the lncRNAs presented.

In addition, 7,895 differentially expressed mRNAs between the RA and $\mathrm{NC}$ groups were identified, which consisted of 4,916 upregulated mRNAs and 2,934 downregulated mRNAs according to the filtering criteria of $\mathrm{P}<0.05$ and fold change $\geq 1.5$. Scatter plot and volcano plot analysis were performed to represent the differentially expressed mRNAs between the two groups, as depicted in Fig. 1B and D. Their distinct expression patterns were also displayed in a hierarchical clustering analysis as presented in Fig. 1F.

GO analysis of the significantly differentially expressed mRNAs indicated that organelle organization, protein modification by small protein conjugation, positive regulation of cytoplasmic mRNA processing, intracellular, intracellular part, intracellular organelle, organelle nucleoplasm and intracellular membrane-bounded organelle were significant terms (Fig. 2). The KEGG analysis of these significantly differentially expressed mRNAs suggested that the cyclic AMP signaling pathway, colorectal cancer, Epstein-Barr virus infection, ErbB signaling pathway, influenza A, proteoglycans in cancer Ras signaling pathway, renal cell carcinoma, sphingolipid signaling pathway and TNF signaling pathway were significant pathways (Fig. S1). Furthermore, according to the KEGG analysis of the significantly differentially expressed lncRNAs, the IL-17 signaling pathway, TNF signaling pathway and intestinal immune network for IgA production were significant (Table SI).
Finally, to identify interactions between mRNAs and IncRNAs, gene co-expression networks were constructed, which were established according to the normalized signal intensity of unit genes. Data were normalized by using the median gene expression value of all transcripts expressed from the same coding gene, without any additional modification of the lncRNA expression value. The differentially expressed IncRNAs and mRNAs were screened from the observed list. For each analyzed gene, the Pearson correlation was calculated and lncRNA-mRNA pairs with significant correlations were selected in order to construct the network. Cytoscape was used to draw the co-expression networks. In the network (Fig. 3), triangles represent the IncRNAs and circles the mRNAs. Red and blue represent up- and downregulated RNAs, respectively. The size of the triangles and circles represents fold changes of lncRNAs and mRNAs with larger sizes indicating higher fold changes. The results revealed a complex lncRNA target network that consisted of 99 matched IncRNA-mRNA pairs. A detailed co-expression network of lncRNAs and mRNAs is provided in Fig. S2.

Validation of differentially expressed lncRNAs. The top six upor downregulated lncRNAs were selected. Of these, four were significant, including an upregulated lncRNA (MIR503HG) and three downregulated IncRNAs (LINC01504, FAM95B1 and LINC01146). Differentially expressed lncRNAs in patients with RA determined by RNA-seq were then verified by RT-qPCR analysis (Fig. 4). The expression of LNC01504, 


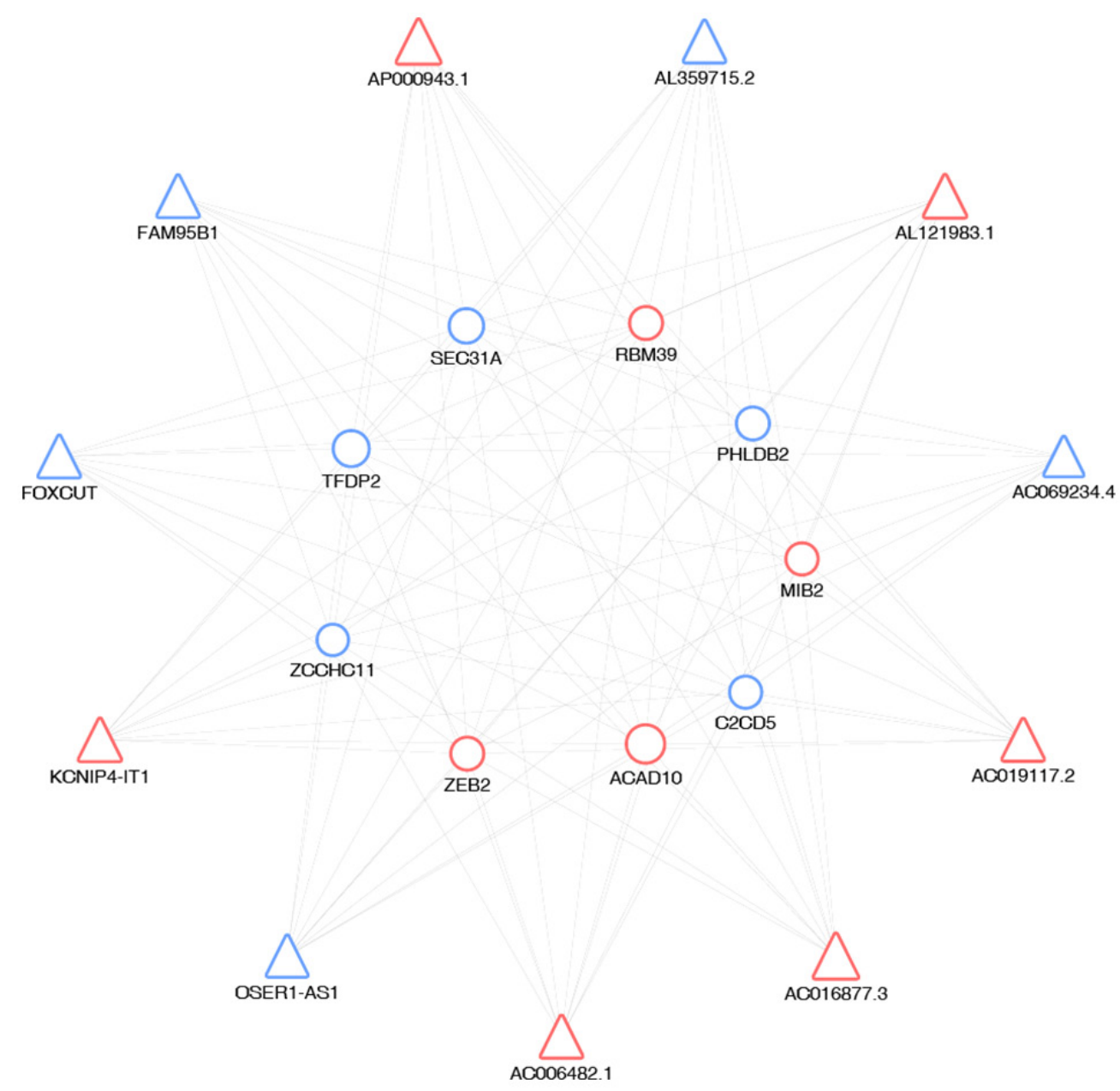

Figure 3. IncRNA-mRNA co-expression network. Triangles represent lncRNAs and circles mRNAs. Red and blue represent up- and down-regulated RNAs, respectively. The size of the triangles and circles indicates the fold change of each RNA. IncRNA, long non-coding RNA.

A

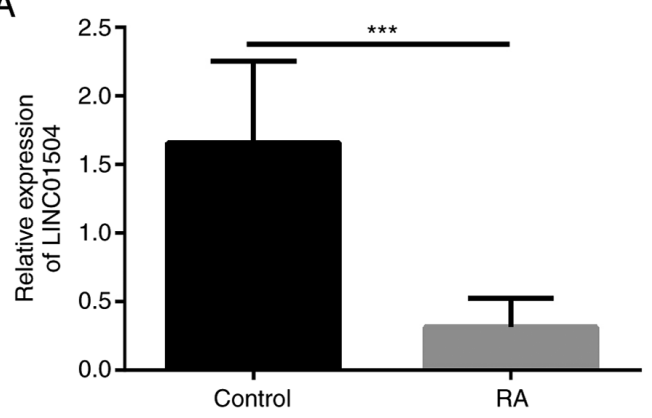

C

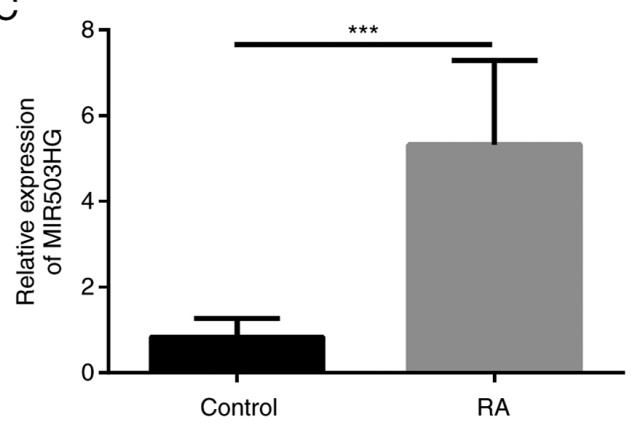

B

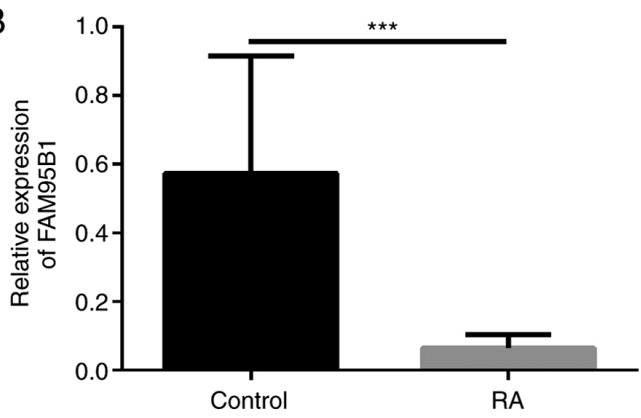

D

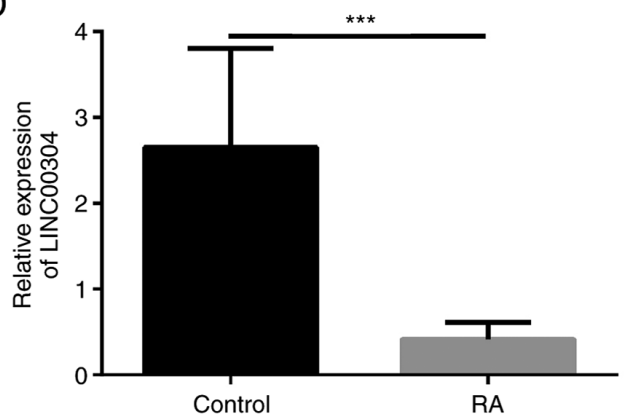

Figure 4. Reverse transcription-quantitative PCR was used to confirm lncRNAs identified to be significantly up- or downregulated in patients with RA compared with healthy controls from the RNA sequencing data. (A) LINC01504; (B) FAM95B1; (C) MIR503HG; and (D) LINC00304. ${ }^{* * *}$ P<0.01. lncRNA, long non-coding RNA; RA, rheumatoid arthritis. 


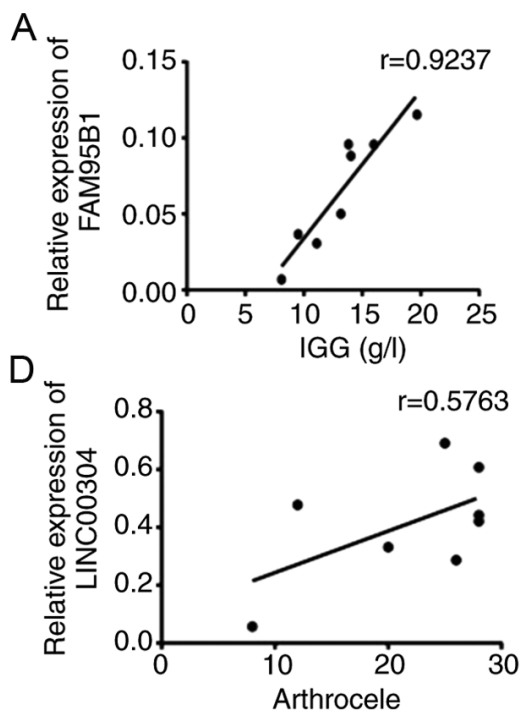

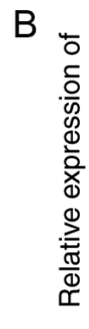
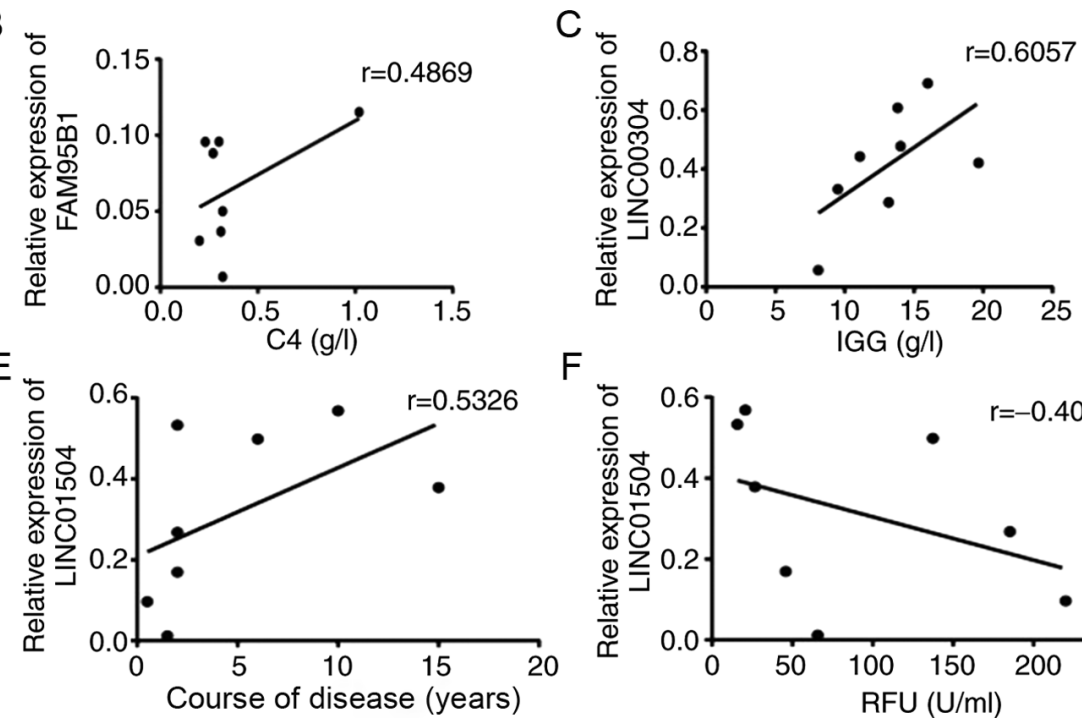

$\mathrm{F}$

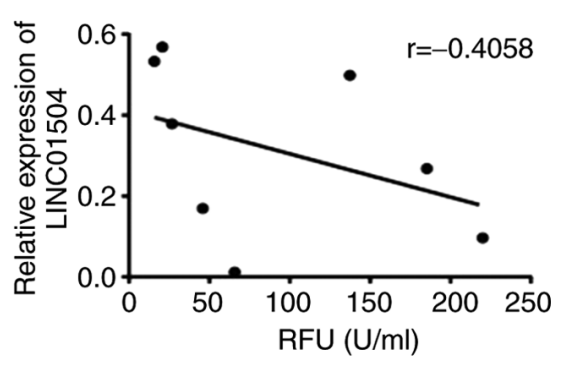

G
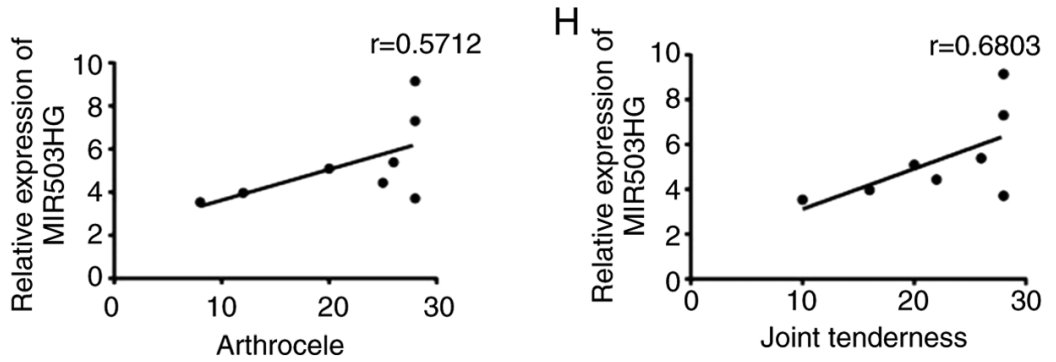

Figure 5. Spearman correlation analysis of clinical variables and confirmed long non-coding RNA in peripheral blood mononuclear cells from patients with rheumatoid arthritis. FAM95B1 was positively correlated with (A) IgG ( $\mathrm{r}=0.9237$; $\mathrm{P}<0.05)$ and (B) $\mathrm{C} 4$ ( $\mathrm{r}=0.4869$; P<0.05). LINC00304 was strongly and positively correlated with (C) IgG ( $\mathrm{r}=0.6057, \mathrm{P}<0.05)$ and $(\mathrm{D})$ arthrocele $(\mathrm{r}=0.5763 ; \mathrm{P}<0.05)$. A strong positive correlation was detected between LINC01504 and $(\mathrm{E})$ course of disease $(\mathrm{r}=0.5326 ; \mathrm{P}<0.05)$. ( F) A negative correlation between LINC01504 and RF was revealed ( $\mathrm{r}=0.4058$; $\mathrm{P}<0.05)$. $(\mathrm{G}) \mathrm{A}$ strong positive correlation between MIR503HG and arthrocele was demonstrated $(\mathrm{r}=0.5712 ; \mathrm{P}<0.05)$. $(\mathrm{H})$ A negative correlation between MIR503HG and joint tenderness was revealed $(\mathrm{r}=0.6083, \mathrm{P}<0.05)$. $\mathrm{C} 4$, complement 4 ; $\mathrm{RF}$, rheumatoid factor.

FAM95B1 and LINC00304 in the control group was higher than that in the RA group and the expression of MIR503HG in the RA group was higher than that in the control group. Spearman tests of the correlation of clinical variables with the lncRNAs in PBMCs from patients with RA were then performed (Fig. 5). According to the results, there was a strong positive correlation of FAM95B1 with $\mathrm{IgG}(\mathrm{r}=0.9237$, $\mathrm{P}<0.05)$ and $\mathrm{C} 4(\mathrm{r}=0.4869, \mathrm{P}<0.05)$, of LINC00304 with $\mathrm{IgG}$ $(\mathrm{r}=0.6057, \mathrm{P}<0.05)$ and arthrocele $(\mathrm{r}=0.5763, \mathrm{P}<0.05)$, of MIR503HG with joint tenderness $(\mathrm{r}=0.6083, \mathrm{P}<0.05)$ and of LINC01504 with course of the disease $(r=0.5326, \mathrm{P}<0.05)$, but a negative correlation between LINC01504 and RF $(\mathrm{r}=0.4058, \mathrm{P}<0.05)$.

\section{Discussion}

RA is a chronic autoimmune joint disease and its persistent inflammation affects bone formation and remodeling, leading to gradual bone destruction (19). Its incidence is high, its pathogenesis is not clear and so far, there is no efficient cure. At present, clinical diagnosis is also difficult. The commonly used imaging and serological tests lack specificity (20). Therefore, the search for specific and early diagnostic molecular markers is of great significance for the early diagnosis and treatment of RA.
It was suggested that during the occurrence and development of RA, lncRNA transcriptional regulation is highly specific, which may be an important feature for the independent prediction of biological indicators (6).

TNF receptor-associated factor 1 (TRAF1) and TRAF1-complement component 5 (TRAF1-C5) are susceptibility genes for the pathogenesis of RA. The level of TRAF1-C5 in the joints of patients with RA increases significantly in the acute phase. On the other hand, the development of arthritis was delayed in an arthritis model of TRAF1-C5-deficient mice. It was indicated that a novel type of 1ncRNA, C5T11ncRNA, was mainly expressed in the nucleus, transcribed from the regions between the TRAF1 and TRAF1-C5 genes in RA patients. There was a positive correlation between C5T1lncRNA and the expression of TRAF1-C5 in synovial fibroblasts and PBMCs in patients with RA. C5T11ncRNA serves a role in the pathogenesis of RA by regulating the transcription of the TRAF1-C5 gene located in the same genomic region (8).

With the completion of the human genome project, it has been indicated that the number of total protein-encoding genes in humans is $<20,000$ (21). Non-coding RNAs refer to a group of RNAs that are not translated into proteins, including small nuclear RNAs, small nucleolar RNAs, microRNAs (miRNAs) and lncRNAs (22). These non-coding RNAs manipulate gene 
expression at the epigenetic, transcription and post-transcriptional level and they also participate in almost all physiologic and pathologic processes (23-25).

lncRNAs influence gene expression at multiple levels and in multiple ways (26). lncRNAs either have roles as transcription factors directly or combine with transcription factors to affect their functions, thereby regulating the expression of associated genes (27). A study revealed that lncRNA Mrhl mediated meiotic commitment of mouse spermatogonial cells by regulating Sox 8 expression (28). Furthermore, lncRNAs act as precursors of miRNAs or as competitive endogenous RNA, which act as molecular sponges to regulate their target gene expression by altering the quantity of miRNA (29). Therefore, lncRNAs engage in the crucial regulation of the biology of various cell types. However, the precise mechanism of action of lncRNAs in RA remains elusive.

In the present study, high-throughput sequencing was used to detect the expression of lncRNA and mRNA in PBMCs of patients with RA and healthy controls. To the best of our knowledge, the present study was the first to analyze the correlation between differentially expressed genes and the clinical laboratory indexes of patients with RA. The results suggested that certain IncRNAs were strongly associated with clinical laboratory indexes. First, the selected differentially expressed lncRNAs were verified by RT-qPCR. A total of two lncRNAs, LINC00968 and MIR503HG, whose expression was upregulated during chondrogenesis, and four of those that were downregulated (LINC01504, FAM95B1, LINC00304 and LINC01146) were selected. Subsequently, the differentially expressed mRNAs were analyzed by GO enrichment and KEGG pathway analysis while the differentially expressed lncRNAs were analyzed by KEGG pathway analysis. The results indicated that for patients with RA, significant changes in their lncRNA expression profile in PBMCs may be related to inflammation. These pathways are related to signaling pathways such as TGF, TNF, EBRr and cAMP, which have been widely confirmed to have an important role in the pathogenesis of RA (30-32).

Subsequently, six differentially expressed lncRNAs were selected for RT-qPCR verification. The expression of LINC00304, LINC01504 and FAM95B1 in the RA group was significantly lower than that in the control group. The expression of MIR503HG in the RA group was significantly higher than that in the control group and the results were consistent with the sequencing. Furthermore, correlations between the expression of LINC00304, LINC01504, FAM95B1 and MIR503HG with biochemical measurements were observed. The results suggested that the levels of FAM95B1 were positively associated with IgG and C4, while the levels of LINC00304 were positively associated with IgG and arthrocele, and there was a positive correlation between MIR503HG and joint tenderness, LINC01504 and course of disease, but a negative correlation between LINC01504 and RF. These results revealed that LINC00304, LINC01504, FAM95B1 and MIR503HG may have critical roles in the pathogenic mechanism of RA.

In RA, as a chronic disease, the presentation and course of the disease differ among individual patients. In recent studies, gene detection in RA has been frequently discussed, but the results were rarely combined with clinical indicators and the patients' quality of life $(33,34)$.
In conclusion, the present study may provide a reference for development of diagnostic tools. However, due to the limitation of the small number of samples, there are still certain deficits and in future studies, a larger cohort will be used to confirm the present results.

\section{Acknowledgements}

Not applicable.

\section{Funding}

The present study was funded by the National Nature Fund Program (grant no. 81973655), The Anhui Key Research and Development Program Foreign Science and Technology Cooperation Project (grant no. 201904b11020011), the Ministry of Science and Technology National Key Research and Development Program Chinese Medicine Modernization Research Key Project (grant no. 2018YFC1705204), the Anhui Provincial Quality Engineering Teaching and Research Project (grant no. 2018jyxm1068), the Key Research and Development Plan Project of Anhui Province (grant no. 201904a07020004), the Anhui Provincial Laboratory of Applied Basis and Development of Internal Medicine of Modern Traditional Chinese Medicine (grant no. 2016080503B041) and the 12th Batch of the '115' Innovation Team of Anhui Province [Anhui Talent Office (2019) No. 1], the Key Laboratory opening Project of Xin'an Ministry of Medical Education (2020xayx08).

\section{Availability of data and materials}

The datasets used and/or analyzed during the current study are available from the corresponding author on reasonable request.

\section{Authors' contributions}

JL, HJ, LW, YS, LX, PZ and DH contributed to the study design. YL and JW contributed to data analysis, wrote the first draft and revised the manuscript. YS, YL, YZ, BB and GS contributed to the specimen and data collection. JL and HJ were the supervisors of the project and contributed to the revision of the manuscript. All authors reviewed and approved the final manuscript.

\section{Ethics approval and consent to participate}

The study complied with the Declaration of Helsinki and was approved by the Institutional Review Board Ethics Committee of The First Affiliated Hospital of Anhui University of Chinese Medicine. Written informed consent was obtained from each patient.

\section{Patient consent for publication}

Not applicable.

\section{Competing interests}

The authors declare that they have no competing interests. 


\section{References}

1. McInnes IB and Schett G: Pathogenetic insights from the treatment of rheumatoid arthritis. Lancet 389: 2328-2337, 2017.

2. Catrina AI, Svensson CI, Malmstrom V, Schett G and Klareskog L: Mechanisms leading from systemic autoimmunity to joint-specific disease in rheumatoid arthritis. Nat Rev Rheumatol 13: 79-86, 2017.

3. Michels AW and Eisenbarth GS: Immunologic endocrine disorders. J Allergy Clin Immunol 125 (Suppl 2): S226-S237, 2010.

4. Sharif K, Sharif A, Jumah F, Oskouian R and Tubbs RS: Rheumatoid arthritis in review: Clinical, anatomical, cellular and molecular points of view. Clin Anat 31: 216-223, 2018.

5. Kowalczyk MS, Higgs DR and Gingeras TR: Molecular biology: RNA discrimination. Nature 482: 310-311, 2012.

6. Muller N, Doring F, Klapper M, Neumann K, Schulte DM, Turk K, Schröder JO, Zeuner RA, Freitag-Wolf S, Schreiber S and Laudes M: Interleukin-6 and tumour necrosis factor-alpha differentially regulate lincRNA transcripts in cells of the innate immune system in vivo in human subjects with rheumatoid arthritis. Cytokine 68: 65-68, 2014.

7. Luo Q, Xu C, Li X, Zeng L, Ye J, Guo Y, Huang Z and Li J: Comprehensive analysis of long non-coding RNA and mRNA expression profiles in rheumatoid arthritis. Exp Ther Med 14: 5965-5973, 2017

8. Messemaker TC, Frank-Bertoncelj M, Marques RB, Adriaans A, Bakker AM, Daha N, Gay S, Huizinga TW, Toes RE, Mikkers HM and Kurreeman F: A novel long non-coding RNA in the rheumatoid arthritis risk locus TRAF1-C5 influences C5 mRNA levels. Genes Immun 17: 85-92, 2016.

9. Moerman RV, Arends S, Mossel E, Kroeze FGM, Vissink A and Bootsma H: 10-year follow-up of patients with rheumatoid arthritis and secondary Sjogren's syndrome or sicca symptoms in daily clinical practice. Clin Exp Rheumatol 38 (Suppl 126) S64-S72, 2020.

10. Sul B, Lee KB, Joo YB, Hong BY, Kim JS, Kim KJ, Park KS, Park YJ and Lim SH: Twelve weeks of strengthening exercise for patients with rheumatoid arthritis: A prospective intervention study. J Clin Med 9: E2792, 2020.

11. Aletaha D, Neogi T, Silman AJ, Funovits J, Felson DT, Bingham CO III, Birnbaum NS, Burmester GR, Bykerk VP, Cohen MD, et al: 2010 Rheumatoid arthritis classification criteria: An American College of Rheumatology/European League Against Rheumatism collaborative initiative. Arthritis Rheum 62: 2569-2581, 2010.

12. Ramírez J, Cuervo A, Celis R, Ruiz-Esquide V, Castellanos-Moreira R, Narváez JA, Gómez-Puerta JA, Pablos JL, Sanmartí R and Cañete JD: Biomarkers for treatment change and radiographic progression in patients with rheumatoid arthritis in remission: A 5 year follow-up study. Rheumatology (Oxford): Jul 12, 2020 (Epub ahead of print). doi: org/10.1093/ rheumatology/keaa258.

13. Wen J, Liu J, Zhang P, Jiang H, Xin L, Wan L, Sun Y, Huang D, Sun Y, Long Y, et al: RNA-seq reveals the circular RNA and miRNA expression profile of peripheral blood mononuclear cells in patients with rheumatoid arthritis. Biosci Rep 40 BSR20193160, 2020.

14. Ju H, Zhang L, Mao L, Wu Y, Liu S, Ruan M, Hu J and Ren G: A comprehensive genome-wide analysis of the long noncoding RNA expression profile in metastatic lymph nodes of oral mucosal melanoma. Gene 675: 44-53, 2018.

15. Yin J, Hu T, Xu L, Li P, Li M, Ye Y and Pang Z: Circular RNA expression profile in peripheral blood mononuclear cells from Crohn disease patients. Medicine (Baltimore) 98: e16072, 2019.

16. Si C, Wang J, Ma W, Hua H, Zhang M, Qian W, Zhou B and Luo D: Circular RNA expression profile in human fibroblast premature senescence after repeated ultraviolet B irradiations revealed by microarray. J Cell Physiol 234: 18156-18168, 2019.
17. Zhao Z, Bai J, Wu A, Wang Y, Zhang J, Wang Z, Li Y, Xu J and Li X: Co-lncRNA: Investigating the lncRNA combinatorial effects in GO annotations and KEGG pathways based on human RNA-Seq data. Database (Oxford) 2015: bav082, 2015.

18. Yang L, Lyu L, Wu W, Lei D, Tu Y, Xu D, Feng J and He L: Genome-wide identification of long non-coding RNA and mRNA profiling using RNA sequencing in subjects with sensitive skin. Oncotarget 8: 114894-114910, 2017.

19. Harnden K, Pease C and Jackson A: Rheumatoid arthritis. BMJ 352: i387, 2016.

20. Pincus T and Sokka T: Laboratory tests to assess patients with rheumatoid arthritis: Advantages and limitations. Rheum Dis Clin North Am 35: 731-734, vi-vii, 2009.

21. ENCODE Project Consortium, Birney E, Stamatoyannopoulos JA, Dutta A, Guigo R, Gingeras TR, Margulies EH, Weng Z, Snyder M, Dermitzakis ET, et al: Identification and analysis of functional elements in $1 \%$ of the human genome by the ENCODE pilot project. Nature 447: 799-816, 2007.

22. Klingenberg M, Matsuda A,Diederichs S and Patel T: Non-coding RNA in hepatocellular carcinoma: Mechanisms, biomarkers and therapeutic targets. J Hepatol 67: 603-618, 2017.

23. Fruci D, Rota R and Gallo A: The Role of HCMV and HIV-1 MicroRNAs: Processing, and mechanisms of action during viral infection. Front Microbiol 8: 689, 2017.

24. Sahni A, Hajjari M, Raheb J, Foroughmand AM and Asgari M: Cloning and over expression of non-coding RNA rprA in E.coli and its resistance to Kanamycin without osmotic shock. Bioinformation 13: 21-24, 2017.

25. Zhang J, Le TD, Liu L and Li J: Inferring miRNA sponge co-regulation of protein-protein interactions in human breast cancer. BMC Bioinformatics 18: 243, 2017.

26. Deniz E and Erman B: Long noncoding RNA (lincRNA), a new paradigm in gene expression control. Funct Integr Genomics 17: 135-143, 2017.

27. Luo G, Liu D, Huang C, Wang M, Xiao X, Zeng F, Wang L and Jiang G: lncRNA GAS5 inhibits cellular proliferation by targeting P27 ${ }^{\mathrm{Kipl}}$. Mol Cancer Res 15: 789-799, 2017.

28. Kataruka S, Akhade VS, Kayyar B and Rao MRS: Mrhl long noncoding RNA mediates meiotic commitment of mouse spermatogonial cells by regulating sox 8 expression. Mol Cell Biol 37: e00632-16, 2017.

29. Laneve P, Po A, Favia A, Legnini I, Alfano V, Rea J, Di Carlo V, Bevilacqua V, Miele E, Mastronuzzi A et al: The long noncoding RNA linc-NeD125 controls the expression of medulloblastoma driver genes by microRNA sponge activity. Oncotarget 8: 31003-31015, 2017

30. Cui Y, Yi Q, Sun W, Huang D, Zhang H, Duan L, Shang H, Wang D and Xiong J: Molecular basis and therapeutic potential of myostatin on bone formation and metabolism in orthopedic disease. BioFactors: Sep 30, 2020 (Epub ahead of print). doi: $10.1002 /$ biof.1675.

31. Cheng Q, Wu H and Du Y: The roles of small-molecule inflammatory mediators in rheumatoid arthritis. Scand J Immunol: Oct 8, 2020 (Epub ahead of print). doi: 10.1111/sji.12982.

32. Wu HX, Chen JY, Wang QT, Sun WY, Liu LH, Zhang LL and Wei W: Expression and function of $\beta$-arrestin 2 stimulated by IL-1 $\beta$ in human fibroblast-like synoviocytes and the effect of paeoniflorin. Int Immunopharmacol 12: 701-706, 2012.

33. Berner C, Erlacher L, Fenzl KH and Dorner TE: A cross-sectional study on self-reported physical and mental health-related quality of life in rheumatoid arthritis and the role of illness perception. Health Qual Life Outcomes 16: 238, 2018.

34. Al-Herz A, Saleh K, Al-Awadhi A, Al-Kandari W, Hasan E, Ghanem A, Hussain M, Ali Y, Nahar E, Alenizi A, et al: Accessibility to biologics and its impact on disease activity and quality of life in patients with rheumatoid arthritis in Kuwait. Clin Rheumatol: Oct, 12, 2020 (Epub ahead of print). doi org/10.1007/ s10067-020-05444-2.

This work is licensed under a Creative Commons Attribution-NonCommercial-NoDerivatives 4.0 International (CC BY-NC-ND 4.0) License. 